where $H$ is a ("homogeneous") set having relative exterior measure 1 at every one of its points, and $Z$ is of measure zero.

We obtain a particular case of our theorem if we assume $A$ to be a measurable set. Exterior measure will then be replaced by measure, and relative exterior measure by "relative measure." We thus have

COROLlary 2. The relative measure of a measurable set is 1 at every one of its points except possibly at those of a set of measure zero.

Corollary 2 is equivalent to a theorem of Lebesgue-Denjoy.* The present note, therefore, also gives a very simple proof of this important theorem.

So far the author has not succeeded in proving the theorem of this note for higher dimensions, although there seems to be little ground for doubting its validity in $n$-space.

UNIVERSITY OF ILLINOIS.

\title{
A GENERAL FORM OF GREEN'S THEOREM.
}

BY PROFESSOR P. J. DANIELL.

In this paper a form of Green's theorem is considered which applies, on the one hand, to the boundary of any set $E$, measurable Borel, and relates, on the other hand, to potential functions which satisfy a general integral form of Poisson's equation,

$$
\int_{B(E)} \frac{\partial V}{\partial n} d s=\int_{E} d \alpha(x, y)
$$

where $\alpha(x, y)$ is some function of limited variation in $(x, y)$. In particular it can be used in mathematical physics in problems in which mass (or electric charge) is not distributed continuously.

Let $V_{1}(x, y), V_{2}(x, y)$ be two potential functions defined and

* Lebesgue, Leçons sur l'Intégration, pp. 123-124, and Denjoy, loc. cit., pp. 132-137. "Relative measure" is equivalent with Denjoy's "épaisseur." Lebesgue's considerations are indirect (as far as the theorem in question is concerned), being based on properties of integrals. Denjoy's proof is direct, but still comparatively involved and long. 
"differentiable"* in the fundamental square $J(0 \leqq x \leqq 1$, $0 \leqq y \leqq 1$ ); let

$$
\frac{\partial V_{1}}{\partial x}=v_{1}, \quad \frac{\partial V_{1}}{\partial y}=-u_{1}, \quad \frac{\partial V_{2}}{\partial x}=v_{2}, \quad \frac{\partial V_{2}}{\partial y}=-u_{2}
$$

be summable with their squares in $J$.

Furthermore assume that $u_{1}, u_{2}$ satisfy $R_{1} ; v_{1}, v_{2}$ satisfy $R_{2}$. $R_{1}$. The total variation of $u(x, y)$ varying $y(0 \leqq y \leqq 1)$ is a function of $x$, finite nearly everywhere and summable in $(0 \leqq x \leqq 1)$.

$R_{2}$. The same as $R_{1}$, with $v$ in place of $u$, and with the rôles of $x$ and $y$ interchanged.

In a previous papert the author has shown that, under these restrictions, we may express

in the form

$$
\int_{B(E)} \frac{\partial V_{1}}{\partial n} d s=\int_{B(E)} u_{1} d x+v_{1} d y
$$

$$
\int_{E} d \alpha_{1}
$$

In the present paper it is further proved that

$$
\int_{B(E)} V_{2} \frac{\partial V_{1}}{\partial n} d s=\int_{E} V_{2} d \alpha_{1}+\int_{E}\left(u_{1} u_{2}+v_{1} v_{2}\right) d x d y .
$$

Consider a rectangle $r(a \leqq x \leqq b, c \leqq y \leqq d)$ contained in $J$. We have

$$
\begin{aligned}
\int_{B(r)} u_{1} d x & =\int_{a}^{b}\left[u_{1}(x, c)-u_{1}(x, d)\right] d x \\
& =-\int_{a}^{b} d x \int_{c}^{d} d_{y} u_{1}(x, y) .
\end{aligned}
$$

In this $\int_{c}^{d} d_{y} u_{1}(x, y)$ may be regarded as a Stieltjes integral with $y$ as variable, which, by $R_{1}$, exists for nearly all values of $x$ and is summable in $x$. Then

$$
-\int_{a}^{b} d x \int_{c} d_{y} u_{1}(x, y)=\int_{r} d \alpha^{\prime}(x, y)
$$

* De la Vallée Poussin, Cours d'Analyse, 3d ed., vol. 1, § 147.

$\uparrow$ P. J. Daniell, this Bulletrn, Nov., 1918. 
will be an absolutely additive function of rectangles $r$. Similarly

$$
\begin{aligned}
\int_{B(r)} v_{1} d y & =\int_{c}^{d}\left[v_{1}(b, y)-v_{1}(a, y)\right] d y \\
& =\int_{c}^{d} d y \int_{a}^{b} d_{x} v_{1}(x, y) \\
& =\int_{r} d \alpha^{\prime \prime}(x, y), \\
\int_{B(r)} u_{1} d x+v_{1} d y & =\int_{r} d \alpha(x, y),
\end{aligned}
$$

where $\alpha=\alpha^{\prime}+\alpha^{\prime \prime}$.

For nearly all values of $x,\left(\partial V_{2} / \partial y\right)=-u$ is uniformly bounded with respect to $y$, by $R_{1}$. Then, for nearly all values of $x, V_{2}(x, y)$ is an absolutely continuous function of $y$ and must be of limited variation in $y$.

By a theorem on integration by parts,*

$$
\begin{aligned}
-\int_{c}^{d} d_{y}\left(V_{2} u_{1}\right) & =-\int_{c}^{d} V_{2} d_{y} u_{1}-\int_{c}^{d} u_{1} d_{y} V_{2} \\
& =-\int_{c}^{d} V_{2} d_{y} u_{1}+\int_{c}^{d} u_{1} u_{2} d y
\end{aligned}
$$

for nearly all values of $x$.

Again since $V_{2}$ is "differentiable" at every point of $J$, it is continuous $\dagger$ and uniformly bounded.

If $\max \left|V_{2}\right|=K$, the total variation of $V_{2} u_{1}$, varying $y(0 \leqq y \leqq 1)$ will be not greater than

$$
K \times \text { variation of } u_{1}+\int_{0}^{1}\left|u_{1} u_{2}\right| d y .
$$

$u_{1}, u_{2}$ are summable with their squares in $J$, so that $u_{1} u_{2}$ is also summable in $J$, or $\int_{0}^{11}\left|u_{1} u_{2}\right| d y$ is a summable function of $x$ where it exists (nearly everywhere). Then $V_{2} u_{1}$ will also satisfy $R_{1}$, and similarly $V_{2} v_{1}$ will satisfy $R_{2}$.

* P. J. Daniell, Transactions Amer. Math. Society, October, 1918.

$\uparrow$ De la Vallée Poussin, ibid. 
Combining the various facts, we then obtain

$$
\begin{aligned}
\int_{B(r)} V_{2} u_{1} d x & =-\int_{a}^{b} d x \int_{c}^{d} d_{y}\left(V_{2} u_{1}\right) \\
& =-\int_{a}^{b} d x \int_{c}^{d} V_{2} d_{y} u_{1}+\int_{a}^{b} d x \int_{c}^{d} u_{1} u_{2} d y \\
& =\int_{r} V_{2} d \alpha^{\prime}+\int_{r} u_{1} u_{2} d x d y .
\end{aligned}
$$

The change from repeated to double integrals is legitimate, in the first integral (Stieltjes) because $V_{2}$ is uniformly continuous, in the second because $u_{1} u_{2}$ is summable in $J$. Similarly

Then

$$
\int_{B(r)} V_{2} v_{1} d y=\int_{r} V_{2} d \alpha^{\prime \prime}+\int_{r} v_{1} v_{2} d x d y .
$$

$$
\int_{B(r)} V_{2}\left(u_{1} d x+v_{1} d y\right)=\int_{r} V_{2} d \alpha_{1}+\int_{r}\left(u_{1} u_{2}+v_{1} v_{2}\right) d x d y .
$$

This is an equation in which all three expressions are absolutely additive functions of rectangles, and $V_{2} u_{1}, V_{2} v_{1}$ satisfy $R_{1}, R_{2}$ respectively; therefore for any set $E$, measurable Borel, contained in $J$,

$$
\int_{B(E)} V_{2} \frac{\partial V_{1}}{\partial n} d s=\int_{E} V_{2} d \alpha_{1}+\int_{E}\left(u_{1} u_{2}+v_{1} v_{2}\right) d x d y .
$$

This was the theorem to be proved and we can rewrite it in the form

$$
\int_{B(E)} V_{2} \frac{\partial V_{1}}{\partial n} d s=\int_{E} V_{2} d \alpha_{1}+\int_{E}\left(\operatorname{grad} V_{1} \cdot \operatorname{grad} V_{2}\right) d S .
$$

Corollary 1. Interchanging $V_{1}, V_{2}$ and subtracting,

$$
\int_{B(E)}\left(V_{2} \frac{\partial V_{1}}{\partial n}-V_{1} \frac{\partial V_{2}}{\partial n}\right) d s=\int_{E} V_{2} d \alpha_{1}-\int_{E} V_{1} d \alpha_{2} .
$$

Corollary 2. Instead of this make $V_{1}=V_{2}=V$.

$$
\int_{B(E)} V \frac{\partial V}{\partial n} d s=\int_{E} V d \alpha+\int_{E} \operatorname{grad}^{2} V d S .
$$


Three dimensions. The case of three dimensions is more valuable in applications, and the proof is exactly similar. We content ourselves with the mere statement. [Our previous notation is altered; $u_{1}$ takes the place of $v_{1}$, and $v_{1}$ of $-u_{1}$.] Let $V_{1}, V_{2}$ be two potential functions, defined and "differentiable" in the cube $J(0 \leqq x \leqq 1,0 \leqq y \leqq 1,0 \leqq z \leqq 1)$; let the six partial derivatives be summable with their squares in $J$; and let $\partial V_{1} / \partial x, \partial V_{2} / \partial x$ satisfy $R_{1} ; \partial V_{1} / \partial y, \partial V_{2} / \partial y$ satisfy $R_{2} ; \partial V_{1} / \partial z, \partial V_{2} / \partial z$ satisfy $R_{3}$.

$R_{1}$. The total variation of $u$ varying $x(0 \leqq x \leqq 1)$ is a function of $(y, z)$ finite nearly everywhere and summable in $(0 \leqq y \leqq 1,0 \leqq z \leqq 1)$.

$R_{2}, R_{3}$. The same as $\bar{R}_{1}$ with $v, w$ in place of $u$ and with cyclical interchanges of $x, y, z$.

If the element of normal is drawn outwards,

$$
\int_{B(r)} \frac{\partial V_{1}}{\partial n} d S=\int_{r} d \alpha_{1}
$$

is an absolutely additive function of rectangular parallelopipeds $r$ and $\alpha_{1}(x, y, z)$ is a function of limited variation in $J$. Then we can define for any set $E$, in $J$, measurable Borel,

$$
\int_{B(E)} \frac{\partial V_{1}^{\top}}{\partial n} d S=\int_{E} d \alpha_{1}
$$

and Green's theorem becomes

$$
\int_{B(E)} V_{2} \frac{\partial V_{1}}{\partial n} d S=\int_{E} V_{2} d \alpha_{1}+\int_{E}\left(\operatorname{grad} V_{1} \cdot \operatorname{grad} V_{2}\right) d \text { vol. }
$$

The two corresponding corollaries follow immediately.

Rice Institute,

Houston, Texas. 